\title{
Metallicity distribution functions using Gaia-DR1 data
}

\author{
Georges Kordopatis ${ }^{1}$ and RAVE collaboration \\ ${ }^{1}$ Université Côte d'Azur, Observatoire de la Côte d'Azur, CNRS, Laboratoire Lagrange, France \\ email: gkordo@oca.eu
}

\begin{abstract}
The metallicity distribution function (MDF) of the stellar components of the Milky Way hold valuable information regarding the processes that have taken place in the evolution of our Galaxy. In this proceeding, we investigate updates concerning the MDF now that the Tycho-Gaia Astrometric Solution (TGAS) catalogue has been released and that trigonometric distances are available. In particular, vertical changes and skewness of the MDF are investigated, together with the properties of the metal-rich stars in the sample, at different positions in the Galaxy.
\end{abstract}

Keywords. Milky Way, stellar content, evolution, metallicity

\section{Introduction}

The advent of large spectroscopic surveys has enabled the possibility to obtain precise abundance measurements of the photospheres for several hundreds of thousands stars, thought to reflect the chemical composition of the interstellar medium in which they were born. As a consequence, information such as the mean, dispersion and skewness of the metallicity distribution functions (MDF) at different regions of the Milky Way hold valuable information regarding the star formation history of those regions, about the presence of stars either not being born where they are being observed, and/or born with migrated or extra-galactic gas, and hence help us to disentangle the evolution of our Galaxy (Bland-Hawthorn \& Gerhard 2016).

Up to recently, the study of the MDF with precise stellar locations (via parallax measurements) for FGK stars could be done only up to a few 100 pc from the Sun, i.e. within the Hipparcos volume. To go beyond this limit, so-called spectroscopic distances projecting on isochrones the stellar atmospheric parameters were used (e.g.: Pont \& Eyer 2004; Kordopatis et al. 2011). Since Septembre 2016, the volume for which we have parallaxes has increased by more than an order of magnitude, thanks to the Gaia space mission (Gaia Collaboration et al. 2016a). The first Gaia data-release (Gaia Collaboration et al. 2016b), and in particular its sub-catalogue TGAS (Tycho-Gaia Astrometric Solution, Lindegren et al. 2016) published 2 million parallaxes and proper motions for stars brighter than the 11th magnitude, allowing to have a better view on both the tails of the MDFs and the way chemistry is linked to the three-dimensional kinematics of stars. In this proceeding, we show updates on that topic. Section 2 describes the datasets that have been combined, Sect. 3 shows some preliminary results, and Sect. 4 presents the perspectives.

\section{Dataset used: the joint RAVE-TGAS catalogue}

Our sample consists of $\sim 2 \cdot 10^{5}$ stars with measured atmospheric parameters, metallicities and radial velocities, coming from the RAVE fifth data release (Kunder et al. 

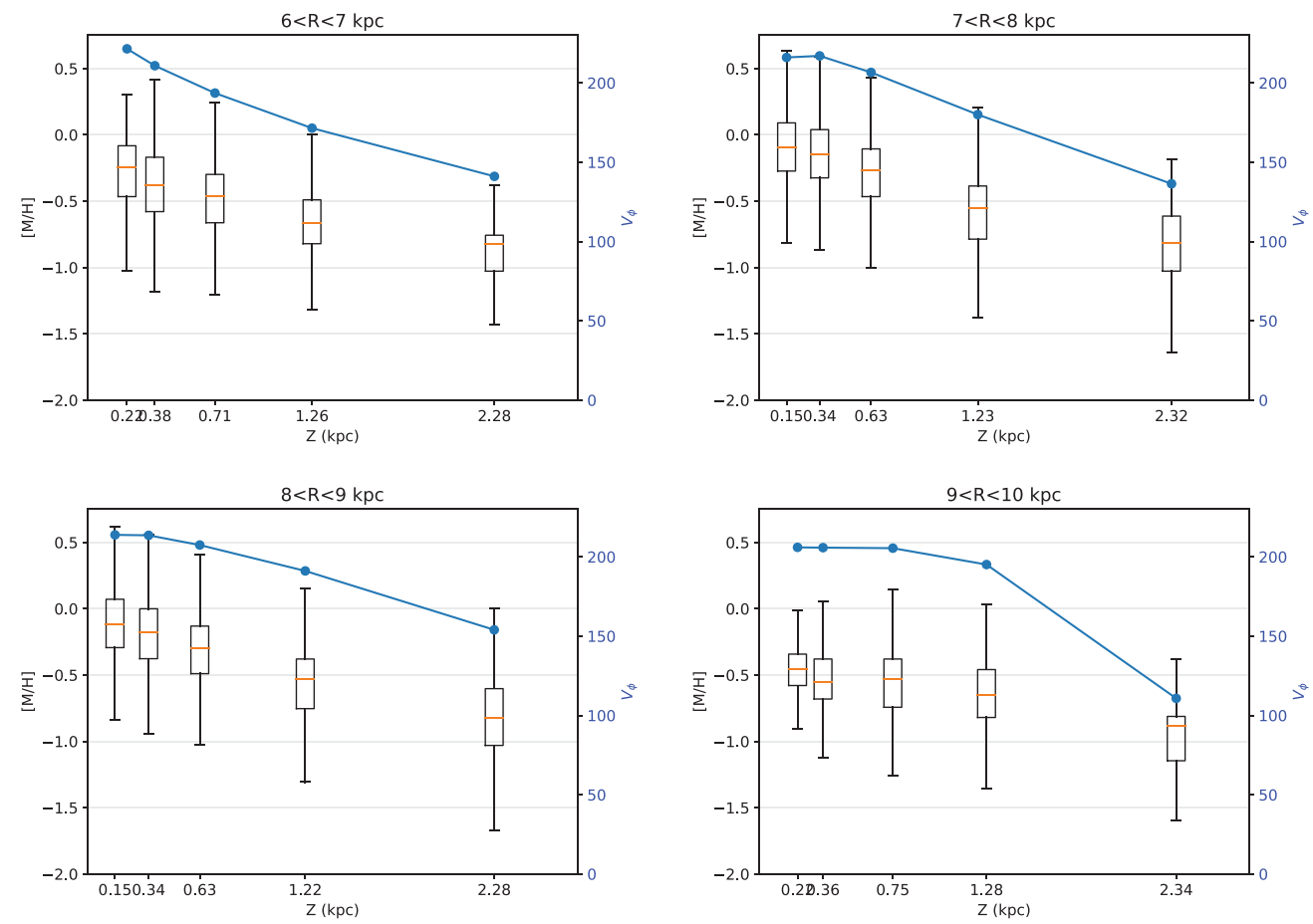

Figure 1. Box plots representing the median metallicity (orange line) as a function of absolute distance from the Galactic plane, $\mathrm{Z}$, for different radial ranges, $\mathrm{R}$, from the Galactic center. The actual boxes enclose the metallicity value of the first and third quartile, whereas the sizes of the bar span 99 per cent of the distribution. The median $V_{\phi}$ value, in $\mathrm{km} \mathrm{s}^{-1}$, for each spatial bin is also represented in blue (and right-hand-side $y$-axis).

2017), combined with UCAC5 proper motions (Zacharias et al. 2017) and improved RAVE spectroscopic distances (McMillan \& RAVE 2017, this volume). The reason for not using the TGAS proper motions or parallaxes is that both UCAC5 and the new RAVE distances build on Gaia's measurements to improve the transverse velocities and distances. In particular, the new spectroscopic distances take into account not only the stellar atmospheric parameters but also Gaia's parallax in order to infer the most likely distance of the observed star (McMillan et al., in prep.). This leads to more reliable distances, in particular for the stars for which the TGAS fractional uncertainty on the parallax is greater than 20 per cent (mostly corresponding to giants in our sample).

\section{Results}

Figure 1 shows box-plots representing the metallicity distributions as a function of height above the Galactic plane, at different Galactic radii (different panels). The $Z$-bins closest to the plane are suffering from selection biases and completeness issues that differ from one $R$-bin to the other and hence should not be used for the analysis, as they are not representative of the underlying population.

Excluding those bins, we measure the following vertical metalicity gradients, $\partial[\mathrm{M} / \mathrm{H}] / \partial|Z|$, for the four $1 \mathrm{kpc}$-wide radial bins: $-0.27,-0.34,-0.33$ and $-0.19 \mathrm{dex} \mathrm{kpc}^{-1}$, going from $6 \mathrm{kpc}$ to $10 \mathrm{kpc}$, respectively. These values are compatible, within the errors (of the order of $0.07 \mathrm{dex} \mathrm{kpc}^{-1}$ ), with the ones of Schlesinger et al. (2014) of $-0.243 \pm 0.05 \mathrm{dex} \mathrm{kpc}^{-1}$, obtained using SEGUEG dwarfs. We note, however, that our measurements should be 


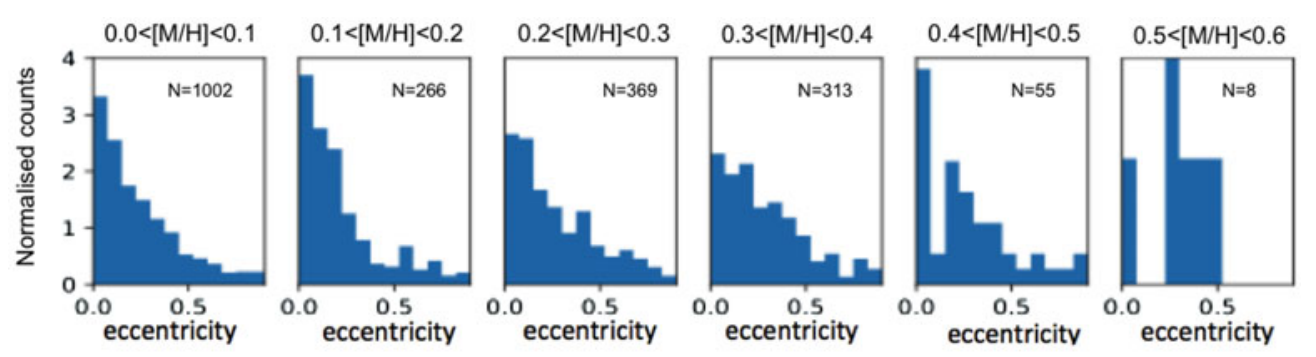

Figure 2. Normalised distribution of stellar eccentricities for the super metal-rich targets located between $7.5 \leqslant R \leqslant 8.5 \mathrm{kpc}$ and $|Z|<0.25 \mathrm{kpc}$.

considered more reliable compared to previous studies, thanks to the improved distances that are being used here.

Regarding the skewness of the the MDFs, no particular changes are noticed as a function of $R$; this is illustrated with the lack of change of the relative position of the median line within a box from one $R$-bin to the other, at a fixed $Z$. This is not in agreement with the inversion of the MDF's skewness highlighted in Hayden et al. (2015) using APOGEE data. Nevertheless, a more thorough analysis, taking into account the completeness of our sample (see Sect. 4), needs to be performed in order to confirm this statement.

We have investigated the impact on the MDFs of the new datasets available by computing similar plots using pure spectroscopic distances and UCAC4 proper motions (in practice, here, we have used the RAVE DR4 catalogue, Kordopatis et al. 2013). We have not found any significant change on the mean of the MDFs. That said, the updated tails of the MDFs make more sense using the Gaia data: many super metal-rich stars $([\mathrm{M} / \mathrm{H}]>0$, noted SMR hereafter) found previously to occupy large distances from the Galactic plane are found to be closer to the disc, whereas metal-poor stars are also shifted to higher $Z$. We note, however, that few SMR stars can still be detected, up to $1 \mathrm{kpc}$ from the Galactic plane, confirming previous studies (see, for example, Kordopatis et al. 2015). The majority of these SMR stars, even though they have updated eccentricities, are found to be in majority on circular orbits ( $e<0.2$, see Fig. 2), therefore having radially migrated from the inner parts of the disc through mechanisms involving co-rotation resonances with the spiral arms (e.g. Sellwood \& Binney 2002).

Finally, it should be highlighted that we find a remarkably flat trend for the mean $V_{\phi}$ as a function of $Z$ for our outermost radial bin $(9<R<10 \mathrm{kpc})$. Up to almost $750 \mathrm{pc}$ from the plane, we find stars to have $\left\langle V_{\phi}>\approx 220 \mathrm{~km} \mathrm{~s}^{-1}\right.$, i.e. associated to a stellar population dominated by the thin disc. This seems to confirm the thin disc flaring and the absence of the thick disc at the outer disc, as already suggested by other studies (Bensby et al. 2011; Minchev et al. 2014; Bovy et al. 2016; Kordopatis et al. 2017), but now clearly indicated with the simple kinematics.

\section{Perspectives}

In this preliminary work, we have combined the stars from the north and the south Galactic cap, and have not corrected for the selection function (noted SF, hereafter) of the sample.

On the one hand, the impact of the RAVE SF should not be dramatic, especially at intermediate and high Galactic latitudes. Indeed, Wojno et al. (2017) has shown that RAVE's SF does not bias the kinematics nor the metallicity distributions, for typical FGK stars under the condition that the stars located close to the Galactic plane (outside the 
RAVE footprint) are removed. On the other hand, the SF of TGAS (Gaia Collaboration et al. 2016b), is more complicated and involves amongst other the scanning law of the satellite (only stars with five astrometric transits are included) and the proper motions of the stars (high proper motion stars are removed). In Kordopatis et al. (in prep.), we combine the Wojno et al. (2017) and Bovy (2017) tools to correct for the selection function and further investigate whether metallicity asymmetries exist relative to the Galactic plane.

\section{Acknowledgements}

This work has made use of data from the European Space Agency (ESA) mission Gaia (https://www.cosmos.esa.int/gaia), processed by the Gaia Data Processing and Analysis Consortium (DPAC, https://www.cosmos.esa.int/web/gaia/dpac/consortium). Funding for the DPAC has been provided by national institutions, in particular the institutions participating in the Gaia Multilateral Agreement. Funding for RAVE has been provided by: the Australian Astronomical Observatory; the Leibniz-Institut für Astrophysik Potsdam (AIP); the Australian National University; the Australian Research Council; the French National Research Agency; the German Research Foundation (SPP 1177 and SFB 881); the European Research Council (ERC-StG 240271 Galactica); the Instituto Nazionale di Astrofisica at Padova; The Johns Hopkins University; the National Science Foundation of the USA (AST-0908326); the W. M. Keck foundation; the Macquarie University; the Netherlands Research School for Astronomy; the Natural Sciences and Engineering Research Council of Canada; the Slovenian Research Agency; the Swiss National Science Foundation; the Science \& Technology Facilities Council of the UK; Opticon; Strasbourg Observatory; and the Universities of Groningen, Heidelberg and Sydney. The research leading to these results has received funding from the European Research Council under the European Union's Seventh Framework Programme (FP7/2007-2013)/ERC grant agreement no. 321067. The RAVE web site is at http://www.rave-survey.org.

\section{References}

Bensby, T., et al., 2011, ApJ, 735, 46

Bland-Hawthorn, J. \& Gerhard, O., 2016, ARA\&A, 54, 561

Bovy, J., et al., 2016, ApJ, 823, 30

Bovy, J., 2017, MNRAS, submitted, arXiv:1704.05063

Gaia Collaboration, Brown, A. G. A., Vallenari, A., et al. 2016, A\& A, 595, A2

Gaia Collaboration, Prusti, T., de Bruijne, J. H. J., et al. 2016, A\&A, 595, A1

Hayden, M., et al., 2015, ApJ, 808, 132

Kordopatis G., et al., 2011, A\& A, 535, A107

Kordopatis G., et al., 2013, AJ, 146, 134

Kordopatis G., et al., 2015, MNRAS, 447, 3526

Kordopatis, G., et al. 2017, MNRAS, 467, 469

Kunder, A., Kordopatis, G., Steinmetz, M., et al. 2017, AJ, 153, 75

Lindegren L., et al., 2016, A\& A, 595, A4

Minchev, I., Chiappini, C., \& Martig, M. 2014, A\&\&A, 572, 92

Pont F., Eyer L., 2004, MNRAS, 351, 487

Sellwood, J. A. \& Binney, J. J., 2002, MNRAS, 336, 785

Schlesinger, K., et al., 2014, ApJ, 791, 112

Wojno, J., Kordopatis, G., Piffl, T., et al. 2017, MNRAS, 468, 3368

Zacharias N., Finch C., Frouard J., 2017, AJ, 153, 166 